(C) 2019 IEEE. Personal use of this material is permitted. Permission from IEEE must be obtained for all other uses, in any current or future media, including reprinting/republishing this material for advertising or promotional purposes, creating new collective works, for resale or redistribution to servers or lists, or reuse of any copyrighted component of this work in other works. 


\section{Uplink Performance of Ultra Dense Networks with Power Control}

\author{
Sinh Cong Lam, Duy Manh Doan, Quoc Tuan Nguyen \\ VNU University of Engineering and Technology, Sdyney \\ Faculty of Electronics and Telecommunications \\ Email: ((congls,tuannq)@vnu.edu.vn
}

\author{
Xiaoying Kong, Kumbesan Sandrasegaran \\ University of Technology, Sdyney \\ Faculty of Engineering and Information Technology \\ Email:(Xiaoying.Kong,Kumbesan.Sandrasegaran)@uts.edu.au
}

\begin{abstract}
In this paper, we study Ultra Dense Networks(UDN) in which the density of BSs is distributed with a density of up to $100 \mathrm{BS} / \mathrm{km}^{2}$. This paper studies the stretched path loss model which recently introduced as an appropriate model for short communications. We introduces a new approach to control the transmit power of the user in which the user's transmit power depends on the distance between the user and its associated Base Station (BS), signal power attenuation. The user performance metric in terms of average coverage probability is mathematically derived. The analytical results indicates that in the case of utilizing power control, increasing the transmit power and the density of BSs can produce negative impacts on the average coverage probability of the user.
\end{abstract}

Index Terms: Poisson Cellular Network, Coverage Probability, Joint Scheduling

\section{INTRODUCTION}

The rapid increase of mobile subscribers as well as data transferred over wireless networks has drawn new requirements for network designer and operators [1]. In that context, Ultra Dense Network (UDNs) has introduced as the new potential network model for next generation of wireless networks, particularly 5G (5 $5^{\text {th }}$ Generation) [2]. In UDN, the Base Stations (BSs) are distributed with an ultra high density, which may be upto $100 \mathrm{BS} / \mathrm{km}^{2}$ [1], to shorten the distance between the user and its serving BS. Figure 1 is an example of UDN in an urban area. Furthermore, the UDN is expected to work at a millimeter band whose frequency is greater than $30 \mathrm{GHz}$.
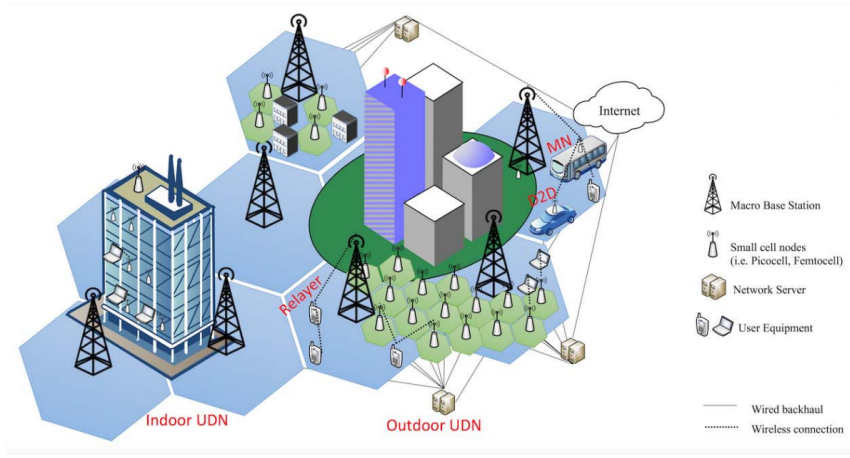

Fig. 1: An example of UDN [3]
With the introduction of UDN, there are a huge number of research work which focus on modeling and performance analysis of this network model [4]. Since the current wellknown propagation path loss models are applied for long distance communications (greater than $1 \mathrm{~km}$ length) such as Okumura model and Hata model [5], or low frequency such as ITU model for the frequency range from $900 \mathrm{MHz}$ to 5.2 $\mathrm{GHz}$ [5]. Thus, stretched path loss model was introduced in Reference [6] as a suitable model for short communications of millimeter wave.

The performance of UDN using stretched path loss model has been investigated in recent research works such as in References [6], [7]. The authors in [6] presented a initial concept of stretched path loss model in which the signal power attenuation over a distance $r$ is $\exp \left(\alpha r^{\beta}\right)$ in which $\alpha$ and $\beta$ are tunable parameters. From the empirical measurements, the authors stated that the selection of $\beta$ depends on the number of obstacles during the transmission line while $\alpha$ represents the effects of these blocks. In Reference [7], the SINR distribution and throughput were presented. However, these works dealt with the downlink in which all the BSs utilize the same transmit power. In this paper, we will focus on the situation of uplink in which each user controls its transmit power to save energy and optimize the system performance.

The uplink performance with power control was studied in the literature such as [8]-[11] in which References [8] and [9] focused on performance analysis and optimization of the fractional frequency reuse technique. The author in Reference [10], [11] discussed about the power control of the user. However, these papers considered the traditional stochastic path loss model which the power loss over the distance $r$ is $r^{-\alpha}$. In this paper, we investigate the power control of the user in the case of stretched path loss model.

The main contributions of this paper are summarized as follows

1) We introduce a new approach to control user's power in the case of UDN with stretched path loss model. The average coverage probability is used throughout the paper as the main user performance metric.

2) A new finding of the network performance trend has been presented. First, the average coverage probability of the user experiences fast decline when the density of BSs 
increases. Secondly, the average coverage probability keeps steadily before passing a fall when the transmit power increases.

\section{NETWORK MODEL}

In this paper, we consider the UDN network in which the BSs are distributed following a Spatial Poisson Point Process (PPP) with a ultra-high density of $\lambda\left(B S / \mathrm{km}^{2}\right)$. The user also distributed according to another PPP with a density of $\lambda^{(u)}$. We assume that $\lambda^{(u)}>>\lambda$, then uses in each cell fully utilized the allocated frequency resource. Hence, each user creates Inter-Cell Interference (ICI) to other users operating on the same uplink frequency band at adjacent cells. If a user does not produce ICI to the user of interest, that user does not effect on the network ICI and will not be considered in this paper.

\section{A. User Power Control}

In the wireless commmunication, the energy resource of the terminal devices is limited, then each mobile user need to control its transmit power to optimize the power consumption as well as network performance. Conventionally, the user transmit power depends on the distance between the user and its serving BS, particularly the signal power loss due to propagation.

We introduce a new path loss model for the user in UDN as follows: The transmit power of the user at distance $r$ from its serving $\mathrm{BS}$ is $P_{0} \exp \left(\alpha r^{\epsilon \beta}\right)$ in which

- $P_{0}$ is the desired uplink received power at the BS.

- $\epsilon$ is the control coefficient $(0<\epsilon<1)$.

- $\alpha$ and $\beta$ are tunable parameters of the stretched path loss model.

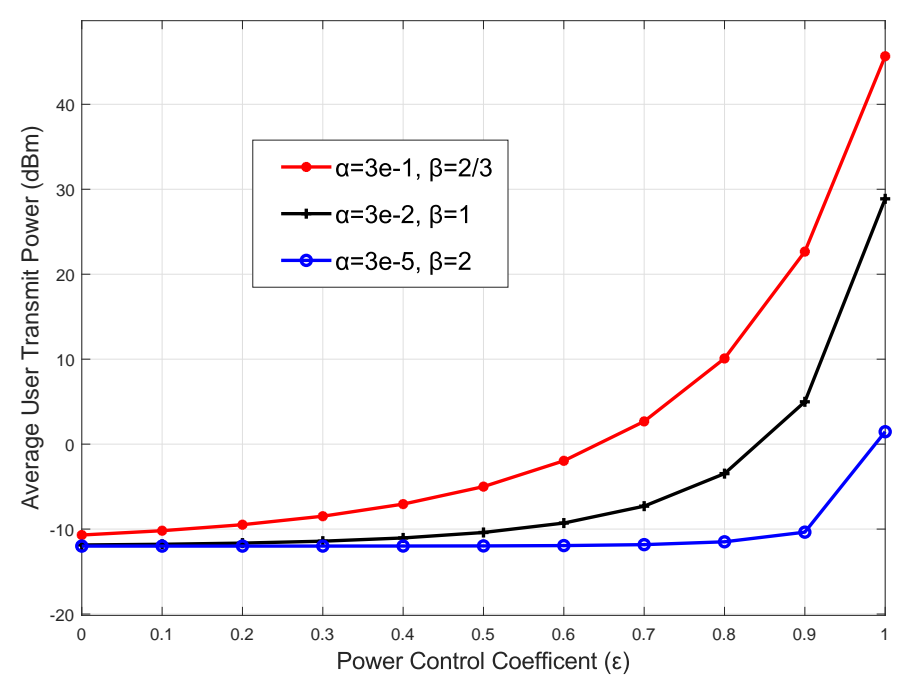

Fig. 2: User Transmit Power with different values of $\epsilon$

Figure 2 presents the transmit power with different values of power control coefficient $\epsilon$. The designed received power at the BS is $P=-12 d \mathrm{Bm}$. It is noted from Reference [6] that $\alpha$ represents the effects of the obstacles on the transmission line. Then when $\alpha$ is set to very small values such as $\alpha=$
$3 \times 10^{-5}$ and $\alpha=3 \times 10^{-2}$, the signal experiences smaller path loss than that does when $\alpha$ receives a greater value of $\alpha=3 \times 10^{-1}$. Thus, in order to obtain the desired received power $P_{0}$ at the BS, the user in environment with $\alpha=3 \times 10^{-1}$ need to transmit at higher power than that in environment with $\alpha=3 \times 10^{-2}$ and $\alpha=3 \times 10^{-5}$.

\section{B. Received SINR at the BS}

We target the typical user which is allocated at the origin and has a distance of $r$ to its serving BS. The set of interfering users to the BS of interest is $\theta$ in which the distance from user $j$ to the BS of interest is $r_{j}$. We denote $d_{j}$ is the distance from interfering user $j$ to its serving BS.

The user associates with the nearest $\mathrm{BS}$, then $r_{j}>d_{j}$. However, Reference [12] proved that the correlation between $r_{j}$ and $d_{j}$ is very weak and can be ignored. Thus, we assume that $r_{j}$ and $d_{j}$ are independent random variables.

The transmit power of user $j$ is $P_{0} \exp \left(-\alpha r^{\epsilon \beta}\right)$. Therefore, we obtain

- The received desired signal power at the typical user is $P_{0} \exp \left(\alpha r^{\epsilon \beta}\right) \exp \left(-\alpha r^{\beta}\right) g$

- The power of interfering signal which is caused by user $j$ is $P_{0} \exp \left(\alpha d_{j}^{\epsilon \beta}\right) \exp \left(-\alpha r_{j}^{\beta}\right) g_{j}$

- The total interfering power at the typical user is

$$
I=\sum_{j \in \theta} P_{0} \exp \left(\alpha d_{j}^{\epsilon \beta}\right) \exp \left(-\alpha r_{j}^{\beta}\right) g_{j}
$$

in which $g$ and $g_{j}$ are channel power gain. Within the content of this paper, they are assumed to be exponential random variables which correspond to the Rayleigh fading environment.

The received SINR at the typical user is obtain by

$$
S I N R=\frac{P_{0} \exp \left(\alpha r^{\epsilon \beta}\right) \exp \left(-\alpha r^{\beta}\right) g}{\sum_{j \in \theta} P_{0} \exp \left(\alpha d_{j}^{\epsilon \beta}\right) \exp \left(-\alpha r_{j}^{\beta}\right) g_{j}+\sigma^{2}}
$$

Since in cellular network, the transmit power of the user is much greater than the power of Gaussian noise, the Gaussian noise can be ignored. Thus the received SINR can be re-written as follows

$$
S I N R=\frac{\exp \left(\alpha r^{\epsilon \beta}\right) \exp \left(-\alpha r^{\beta}\right) g}{\sum_{j \in \theta} \exp \left(\alpha d_{j}^{\epsilon \beta}\right) \exp \left(-\alpha r_{j}^{\beta}\right) g_{j}}
$$

\section{Average Coverage Probability}

The average coverage probability is defined as the probability in which the received SINR at the BS is strong enough for successful data transmission. Denote $T$ is the required SINR for the BS to successfully decode the received signal. Thus, the average coverage probability can be formulated as the following equation:

$$
\mathcal{P}(\epsilon, \lambda)=\mathbb{P}(S I N R>T)
$$


Substituting the definition of SINR in Equation 3 into Equation 4 , we obtain

$$
\begin{aligned}
\mathcal{P}(\epsilon, \lambda) & =\mathbb{P}\left(\frac{\exp \left(\alpha r^{\epsilon \beta}\right) \exp \left(-\alpha r^{\beta}\right) g}{\sum_{j \in \theta} \exp \left(\alpha d_{j}^{\epsilon \beta}\right) \exp \left(-\alpha r_{j}^{\beta}\right) g_{j}}>T\right) \\
& =\mathbb{P}\left(g>T \frac{\sum_{j \in \theta} \exp \left(\alpha d_{j}^{\epsilon \beta}\right) \exp \left(-\alpha r_{j}^{\beta}\right) g_{j}}{\exp \left(\alpha r^{\epsilon \beta}\right) \exp \left(-\alpha r^{\beta}\right)}\right)
\end{aligned}
$$

Since $g$ and $g_{j}$ have an exponential distributions whose the Cumulative Density Function $F_{G}(x)=\exp (-g)$, then $\mathcal{P}(\epsilon, \lambda)$ equals

$$
\begin{aligned}
& \mathbb{E}\left(g>T \frac{\sum_{j \in \theta} \exp \left(\alpha d_{j}^{\epsilon \beta}\right) \exp \left(-\alpha r_{j}^{\beta}\right) g_{j}}{\exp \left(\alpha r^{\epsilon \beta}\right) \exp \left(-\alpha r^{\beta}\right)}\right) \\
= & \mathbb{E}\left[\exp \left(-T \frac{\sum_{j \in \theta} \exp \left(\alpha d_{j}^{\epsilon \beta}\right) \exp \left(-\alpha r_{j}^{\beta}\right) g_{j}}{\exp \left(\alpha r^{\epsilon \beta}\right) \exp \left(-\alpha r^{\beta}\right)}\right)\right] \\
= & \mathbb{E}\left[\prod_{j \in \theta} \mathbb{E}_{g}\left[\exp \left(-T \frac{\exp \left(\alpha d_{j}^{\epsilon \beta}\right) \exp \left(-\alpha r_{j}^{\beta}\right) g_{j}}{\exp \left(\alpha r^{\epsilon \beta}\right) \exp \left(-\alpha r^{\beta}\right)}\right)\right]\right]
\end{aligned}
$$

Using the properties of the Moment Generating Function of exponential random variables which is $E\left[e^{-s g}\right]=\frac{1}{1+s}$, then we obtain

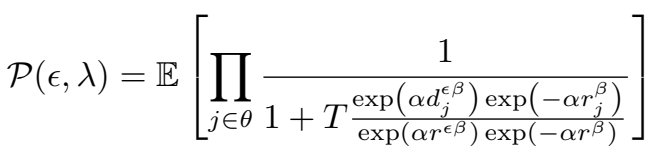

Since $d_{j}$ is the nearest distance from the user $j$ and its serving BS, the Probability Density Function of $d_{j}$ is $f_{D}(x)=$ $2 \pi \lambda x \exp \left(-\pi \lambda x^{2}\right)$. It is also reminded that $d_{j}$ and $d_{k}$ are two independent random variables. Hence, taking the expected value with respects to $d_{j}$, we obtain

$$
\mathcal{P}(\epsilon, \lambda)=\mathbb{E}\left[\prod_{j \in \theta} \int_{0}^{\infty} \frac{2 \pi \lambda d_{j} \exp \left(-\pi \lambda d_{j}^{2}\right)}{1+T \frac{\exp \left(\alpha d_{j}^{\epsilon \beta}\right) \exp \left(-\alpha r_{j}^{\beta}\right)}{\exp \left(\alpha r^{\epsilon \beta}\right) \exp \left(-\alpha r^{\beta}\right)}} d d_{j}\right]
$$

For simple presentation, we denote

$$
\Gamma\left(r, r_{j}\right)=\int_{0}^{\infty} \frac{2 \pi \lambda d_{j} \exp \left(-\pi \lambda d_{j}^{2}\right)}{1+T \frac{\exp \left(\alpha d_{j}^{\epsilon \beta}\right) \exp \left(-\alpha r_{j}^{\beta}\right)}{\exp \left(\alpha r^{\epsilon \beta}\right) \exp \left(-\alpha r^{\beta}\right)}} d d_{j}
$$

Employing the properties of Probability Generating Function, $\mathcal{P}(\epsilon, \lambda)$ is equal to

$$
\mathbb{E}\left[\exp \left(\int_{r}^{\infty}-\pi \lambda r_{j}\left(1-\Gamma\left(r, r_{j}\right)\right) d r_{j}\right)\right]
$$

Taking the expected value with respects to $r$, the average coverage probability $\mathcal{P}(\epsilon, \lambda)$ is given by the following equation

$$
\begin{aligned}
2 \pi \lambda \int_{0}^{\infty} r \exp \left(-\int_{r}^{\infty} \pi \lambda r_{j}\left(1-\Gamma\left(r, r_{j}\right)\right)\right. & \left.d r_{j}\right) \\
& \times \exp \left(-\pi \lambda r^{2}\right) d r
\end{aligned}
$$

Employing changes of variable $r_{j}=y r$ and then $t=\pi \lambda r^{2}$, the expression of $\mathcal{P}(\epsilon, \lambda)$ can be simplified as

$$
\int_{0}^{\infty} \exp \left(-t-t \int_{1}^{\infty}\left(1-y \Gamma\left(\sqrt{\frac{t}{\pi \lambda}}, y \sqrt{\frac{t}{\pi \lambda}}\right)\right) d y\right) d t
$$

Consequently, $\mathcal{P}(\epsilon, \lambda)$ is given by

$$
\mathcal{P}(\epsilon, \lambda)=\frac{1}{1+\int_{1}^{\infty}\left(1-y \Gamma\left(\sqrt{\frac{t}{\pi \lambda}}, y \sqrt{\frac{t}{\pi \lambda}}\right)\right) d y}
$$

Equation 8 gives the relationships between parameters of networks as well as transmission environment and the average coverage probability of the user. Here is the main result of this paper.

a) Special case: No power control $\epsilon=0$.

When $\epsilon=0$, the transmit power of users are the same at $P_{0} \exp (\alpha)$. Thus, $\Gamma\left(r, r_{j}\right)$ in Equation 7 can be re-written as follows

$$
\Gamma\left(r, r_{j}\right)=\int_{0}^{\infty} \frac{2 \pi \lambda d_{j} \exp \left(-\pi \lambda d_{j}^{2}\right)}{1+T \frac{\exp \left(-\alpha r_{j}^{\beta}\right)}{\exp \left(-\alpha r^{\beta}\right)}} d d_{j}
$$

Since $\int_{0}^{\infty} 2 \pi \lambda d_{j} \exp \left(-\pi \lambda d_{j}^{2}\right) d d_{j}=1$,

$$
\Gamma\left(r, r_{j}\right)=\frac{1}{1+T \frac{\exp \left(-\alpha r_{j}^{\beta}\right)}{\exp \left(-\alpha r^{\beta}\right)}}
$$

Substituting $\Gamma\left(r, r_{j}\right)$ into Equation 8 , we obtain the average coverage probability expression as the well-known result in Reference [6].

b) Approximate $\Gamma\left(r, r_{j}\right)$ : .

Employing a change of variable $y=\pi \lambda r^{2}, \Gamma\left(r, r_{j}\right)$ in Equation 7 can be re-written as follows

$$
\Gamma\left(r, r_{j}\right)=\int_{0}^{\infty} \frac{\exp (-y)}{1+T \exp \left(-\alpha r_{j}^{\beta}\right) \exp \left(\alpha(y / \pi \lambda)^{\beta / 2}\right)} d y
$$

The above equation has a suitable form for Gauss-Laguerre quadrature, then $\Gamma\left(r, r_{j}\right)$ can be approximated by

$$
\Gamma\left(r, r_{j}\right) \approx \sum_{i=1}^{n} \frac{w_{i}}{1+T \exp \left(-\alpha r_{j}^{\beta}\right) \exp \left(\alpha\left(x_{i} / \pi \lambda\right)^{\beta / 2}\right)}
$$

in which $w_{i}$ and $x_{i}$ are the weight and root of the Laguerre polynomial with a order of $n$.

\section{Simulation And Discussion}

In this section, we do Monte Carlo simulation to verify the analytical results and visualize the relationship between the power control coefficient $\epsilon$ and density of BSs $\lambda$ with average coverage probability of the user. In the analysis and simulation, the coverage threshold $T$ is set to $T=-3 \mathrm{~dB}$ which means that the user is under the network coverage if the desired received signal power at the BS is at least a half of the total power of interfering signals. 


\section{A. Effects of power control coefficient $\epsilon$}

It is reminded that the environment with $\alpha=3 \times 10^{-1}, \beta=$ $2 / 3$ suffers the strongest power loss, while other with $\alpha=$ $3 \times 10^{-5}, \beta=2$ experiences the lowest power loss. However, it is very interesting from Figure 3 that the user in environment with $\alpha=3 \times 10^{-1}, \beta=2 / 3$ can achieve highest user performance. This performance trend can be explained as follows:

- For the stretched path loss model, i.e. $\exp \left(-\alpha r^{\beta}\right)$ and with three cases of $\alpha, \beta$, e.g. $\left(\alpha=3 \times 10^{-1}, \beta=2 / 3\right)$, $\left(\alpha=3 \times 10^{2}, \beta=1\right)$ and $\left(\alpha=3 \times 10^{-5}, \beta=2\right)$, when the distance $r$ increases and $r>1$, the signal power will have a fastest decline in the case of $\alpha=3 \times 10^{-1}, \beta=2 / 3$ and slowest decline in the case of $\alpha=3 \times 10^{-5}, \beta=2$.

- The desired signal and interfering signals experiences the same path loss model. Thus, the total power of interfering signals in the case of $\beta=2 / 3$ decreases faster than that in the case of $\alpha=3 \times 10^{-1}, \beta=2 / 3$. Meanwhile variance of the desired signal may not be significant. Consequently, the user in the environment with $\alpha=3 \times 10^{-1}, \beta=2 / 3$ can obtain the highest performance, particularly average coverage probability.

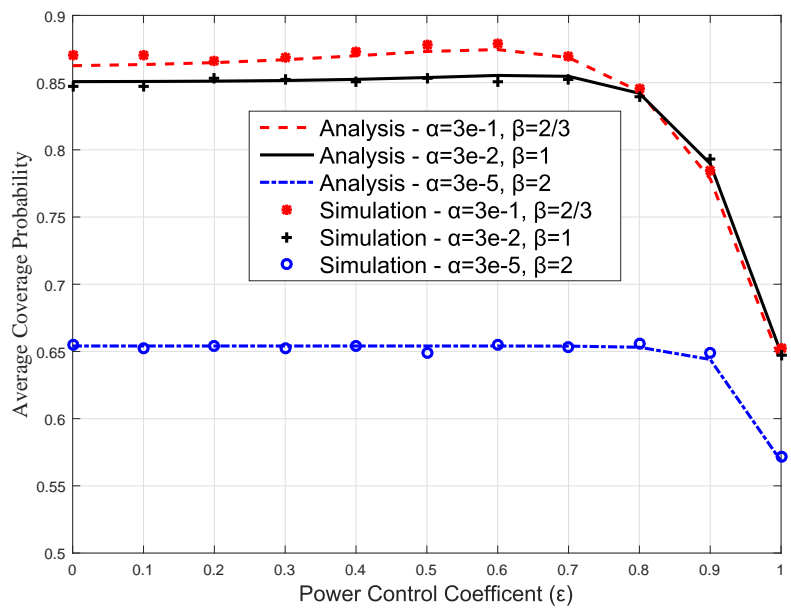

Fig. 3: Effects of power control coefficient on the average coverage probability

Furthermore, Figure 3 also indicates that when the power control coefficient $\epsilon$ increases from 0 to 0.8 , the average coverage probability of the user has a very small changes. Meanwhile when the $\epsilon$ increases from 0.9 to 1 , the user average coverage probability falls. The phenomenon can be explained as follows:

- The transmit power of the user increases with the power control coefficient $\epsilon$ as shown in Figure 3.

- When $\epsilon$ varies between 0 and 0.7 , the user transmit power has a slight change, then there is a balance between the increases of the desired signal power and the interfering signals' power. Hence, the average coverage probability is seem to be constant in this period of $\epsilon$.
- When $\epsilon$ is greater than 0.8 , the user transmit power dramatically increases. That leads to the loss of the balance state and the user performance falls.

\section{B. Effects of density of $B S s \lambda$}

In Figure 4, we study the effects of the density of BSs on the average coverage probability of the user. Similarly to the Figure 3, the user in the environment with $\alpha=3 \times 10^{-1}, \beta=$ $2 / 3$ achieves the highest average coverage probability.

Another interesting fact from Figure 4 that the average coverage probability continuously reduces when the density of BSs increases for all three cases of $\alpha, \beta$. This finding contradict to the results for downlink without the power control in Reference [6].

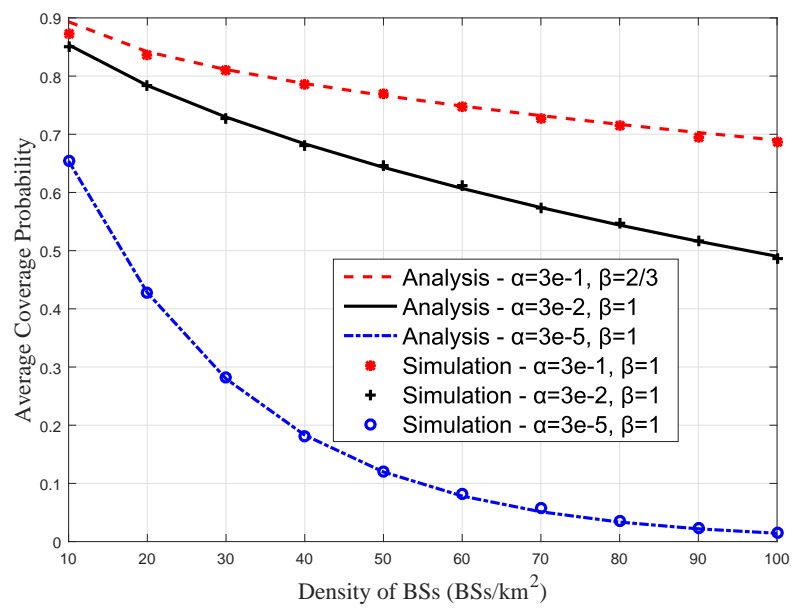

Fig. 4: Effects of density of BSs the average coverage probability

This finding is very valuable for the network design because it indicates that increasing may not improve the user performance in uplink.

\section{Conclusion}

In this paper, we introduced an approach to control the transmit power of the user in UDN, which depends on the power loss of the signal over the transmission line. We derived the user performance in term of average coverage probability expression. Throughout the analytical and simulation results, some interesting findings were found: $(i)$ when the transmit power of the user increases according to the proposed power control model, the average coverage probability of the user keeps at a steady value before passing a fall. (ii) when the density of the BSs increases, the average coverage probability continuously reduces. These findings can be utilized for the network designers.

\section{REFERENCES}

[1] Cisco, "Cisco visual networking index: Global mobile data traffic forecast update, 2015 ? 2020," 2016.

[2] P. Pirinen, "A brief overview of $5 \mathrm{~g}$ research activities," in 1st International Conference on $5 G$ for Ubiquitous Connectivity, Nov 2014, pp. $17-22$.

[3] W. Yu, H. Xu, H. Zhang, D. Griffith, and N. Golmie, "Ultra-dense networks: Survey of state of the art and future directions," in 2016 25th International Conference on Computer Communication and Networks (ICCCN), Aug 2016, pp. 1-10. 
[4] M. Kamel, W. Hamouda, and A. Youssef, "Ultra-dense networks: A survey," IEEE Communications Surveys Tutorials, vol. 18, no. 4, pp. 2522-2545, Fourthquarter 2016.

[5] J. S. Seybold, Introduction to RF Propagation. John Wiley \& Sons, 9 September 2005.

[6] A. AlAmmouri, J. G. Andrews, and F. Baccelli, "Sinr and throughput of dense cellular networks with stretched exponential path loss," IEEE Transactions on Wireless Communications, vol. 17, no. 2, pp. 11471160, Feb 2018

[7] A. AlAmmouri, J. G. Andrews, and F. Baccelli, "Sinr and throughput of dense cellular networks with stretched exponential path loss," IEEE Transactions on Wireless Communications, vol. 17, no. 2, pp. 11471160, Feb 2018.

[8] F. J. Martin-Vega, F. J. Lopez-Martinez, G. Gomez, and M. C. AguayoTorres, "Multi-user coverage probability of uplink cellular systems: A stochastic geometry approach," in 2014 IEEE Global Communi. Conf. (GLOBECOM), Dec 2014, pp. 3989-3994.

[9] H. B. Chang and I. Rubin, "Optimal downlink and uplink fractional frequency reuse in cellular wireless networks," IEEE Trans. on Veh. Tech., vol. 65, no. 4, pp. 2295-2308, April 2016.

[10] M. Ding, P. Wang, D. Lopez-Perez, G. Mao, and Z. Lin, "Performance impact of los and nlos transmissions in dense cellular networks," IEEE Transactions on Wireless Communications, vol. 15, no. 3, pp. 23652380, March 2016.

[11] T. D. Novlan, H. S. Dhillon, and J. G. Andrews, "Analytical modeling of uplink cellular networks," IEEE Trans. Wireless Commun., vol. 12, no. 6, pp. 2669-2679, June 2013.

[12] T. D. Novlan and J. G. Andrews, "Analytical Evaluation of Uplink Fractional Frequency Reuse," IEEE Trans. Commun., vol. 61, no. 5, pp. 2098-2108, May 2013. 